\title{
On the Existence and Robustness of Steady Position-Momentum Correlations for Time-Dependent Quadratic Systems
}

\author{
M. Gianfreda and G. Landolfi \\ Dipartimento di Matematica e Fisica "Ennio De Giorgi", Universitá del Salento and INFN, Sezione di Lecce, \\ 73100 Lecce, Italy \\ Correspondence should be addressed to G. Landolfi, giulio.landolfi@le.infn.it
}

Received 17 October 2011; Revised 10 January 2012; Accepted 13 February 2012

Academic Editor: Ricardo Weder

Copyright (C) 2012 M. Gianfreda and G. Landolfi. This is an open access article distributed under the Creative Commons Attribution License, which permits unrestricted use, distribution, and reproduction in any medium, provided the original work is properly cited.

\begin{abstract}
We discuss conditions giving rise to stationary position-momentum correlations among quantum states in the Fock and coherent basis associated with the natural invariant for the one-dimensional time-dependent quadratic Hamiltonian operators such as the Kanai-Caldirola Hamiltonian. We also discuss some basic features such as quantum decoherence of the wave functions resulting from the corresponding quantum dynamics of these systems that exhibit no timedependence in their quantum correlations. In particular, steady statistical momentum averages are seen over welldefined time intervals in the evolution of a linear superposition of the basis states of modified exponentially damped mass systems.
\end{abstract}

\section{Introduction}

Time-dependent quadratic Hamiltonians describe a number of systems of relevant physical interest in several distinguished areas, ranging from quantum optics [1-6] to cosmology [7-14] and accelerator physics [15]. These Hamiltonians basically represent a paradigm as regards to aspects pertinent to the formal treatment of nonautonomous systems both at classical and at quantum levels (see, e.g. [16-24] and references therein). Several studies have been devoted to providing analytical details of the loss of coherence experienced by wave packets undergoing quantum dynamics ruled by time-dependent quadratic Hamiltonians. In this communication, we present arguments on aspects connected to the requirements on evolving wave packets undergoing time-dependent quadratic Hamiltonian dynamics that exhibit no time dependence in their quantum correlations (and/or the Heisenberg uncertainty relation) among position and momentum operators. To our knowledge, such arguments raised in devising wave packets that satisfy these requirements have never been previously presented. 
Indeed, finding time intervals over which quantum statistical momenta are steady might be perceived as a somewhat surprising result. If so, one could have underestimated lessons coming from one elementary example, namely, the exponentially damped mass (or KanaiCaldirola) systems [25, 26].

The aim of the paper is twofold. First, by resorting to simple examples, we establish that the above-mentioned features are compatible with the rich phenomenology derived from these time-dependent quadratic systems. Second, we explicitly detail some of the statistical features displayed by systems that are time-diffeomorphic to Kanai-Caldirola systems.

The outline of the paper is as follows. Section 2 reviews the Fock states associated with a harmonic oscillator Hamiltonian with time-dependent mass and frequency and the associated invariant operator and creation/annihilation operators. This section proceeds to the Lewis-Riesenfeld (LR) states and the relationship between mass and frequency that gives rise to constant position-momentum correlations. Section 3 uses the mass-frequency relation of Section 2 to find the coefficients of the Bogolubov mapping of the Schrödinger operators into each other and then shows the evolution of the system under time-dependent mass and frequency. In Section 4, the LR geometric phases are introduced to the basis states of the system, and correlation and coherence are discussed. Last section then discusses the generally nonadiabatic nature of a system with constant position momentum correlation.

\section{Fock- and Coherent-Type States Attaining Constant Momenta}

As discussed in numerous papers, the description of the quantum dynamics of timedependent quadratic Hamiltonian systems can be essentially based on adopting a one-mode squeezing formalism. The result, which was first derived by Lewis and Riesenfeld in [18], and excellently reviewed in [19], is well understood from symmetry analysis and invariant theory [27]. In describing nonautonomous systems at the quantum level, it is helpful to know invariant operators [22]. Once the spectral problem for the invariant is solved, each of the resulting eigenvectors is equipped with proper geometric phase factors to represent a particular solution of the time-dependent Schrödinger equation. In particular, consider a general nonautonomous quadratic quantum system governed by the Hamiltonian operator

$$
\widehat{H}=\frac{\widehat{p}^{2}}{2 m}+\frac{1}{2} m \omega^{2} \widehat{q}^{2}
$$

where $\hat{q}, \hat{p}$ are the position and momentum Hamiltonian conjugate operators, respectively, and the mass $m=m(t)$ and frequency $\omega=\omega(t)$ parameters are functions of time $t$. The basic invariant operator

$$
\widehat{I}=\left[\frac{\sigma}{\sqrt{m}} \widehat{p}-\widehat{q} \sigma \sqrt{m} \frac{d}{d t} \ln \frac{\sigma}{\sqrt{m}}\right]^{2}+\frac{m}{4 \sigma^{2}} \widehat{q}^{2}
$$

is then found for the system, with $\sigma=\sigma(t)$ a real positive function that is the solution to the differential equation of the Ermakov type

$$
\frac{d^{2} \sigma}{d t^{2}}+\left(\omega^{2}-\frac{1}{2} \frac{d M}{d t}-\frac{M^{2}}{4}\right) \sigma=\frac{1}{4 \sigma^{3}}, \quad M=\frac{1}{m} \frac{d m}{d t}
$$


(see, e.g. [27, 28]). The function $\sigma$ has a precise meaning. The quantity $\ell=\sigma / \sqrt{m}$ plays the role of a (normalized) time-dependent amplitude of the quasinormal mode solution to the classical equation of motion for the classical counterpart of the system (2.1). A factored expression can be derived that involves time-dependent annihilation and creation operators $\widehat{a}$ and $\hat{a}^{\dagger}$,

$$
\widehat{I}-\frac{\hbar}{2}=\widehat{a}^{\dagger}(t) \widehat{a}(t) \equiv \widehat{N}(t)
$$

( $\left[\widehat{a}(t), \widehat{a}^{\dagger}(t)\right]=1$ and $\widehat{N}(t)$ is the number operator). These ladder operators are linked to position and momentum operators [27]. For later convenience, we define these relations here:

$$
\widehat{q}=\sqrt{\hbar} \ell\left(\widehat{a}+\widehat{a}^{\dagger}\right), \quad \hat{p}=(\Omega \widehat{a}+\text { h.c. }),
$$

with

$$
\Omega=\frac{\sqrt{\hbar}}{2 \ell}(\delta-i), \quad \delta=2 \sigma^{2} \frac{d}{d t} \ln \ell, \quad \ell=\frac{\sigma}{\sqrt{m}}
$$

(equiv., $\delta=m \ell^{2}(d / d t) \ln \ell^{2}$ ). Consequently, Fock-type spaces are defined at different times in terms of the eigenstates $|n\rangle$ of the number operator $\widehat{N}$ (hence of $\widehat{I}$ ), $\widehat{N}|n\rangle=n|n\rangle$. These spaces are then mapped one into the other through a time-dependent Bogolubov transformation [29].

Similarly, coherent-like bases at different times may be defined by means of the eigenstates $|\alpha\rangle$ associated with the spectral problem for the time-dependent operator $\hat{a}$, that is, $\widehat{a}|\alpha\rangle=\alpha|\alpha\rangle$. These are the so-called LR coherent states [18] (actually squeezed states [30]). The number-type states $|n\rangle$, and consequently the coherent-type states a lá Lewis-Riesenfeld $|\alpha\rangle$, do not solve the Schrödinger equation for the Hamiltonian operator (2.1). For this to happen, each $|n\rangle$ has to be equipped with a proper phase (the Lewis-Riesenfeld geometric phase [18], see (4.1) below). This clarifies in several respects why these states are of interest.

Under the time-dependent quadratic Hamiltonian dynamics, physical states (for instance, those that might exhibit minimum Heisenberg uncertainty at some initial time), generally loose coherence during their time evolution as a consequence of the time-dependent Bogolubov (squeezing) transformation. This affects position-momentum correlations. In particular, it turns out that $\delta$ introduced above, (2.6), can be recognized as twice the positionmomentum correlation among LR states because ( $\hbar=1$ hereinafter)

$$
\Delta_{\alpha} \widehat{q} \Delta_{\alpha} \widehat{p}=\frac{1}{2} \sqrt{1+\delta^{2}}, \quad \Delta_{n} \widehat{q} \Delta_{n} \widehat{p}=\left(n+\frac{1}{2}\right) \sqrt{1+\delta^{2}}
$$

(lower-scripts $\alpha$ and $n$ mean that variances are evaluated in the coherent or Fock basis, resp.). Position-momentum correlations among eigenvectors of the time-dependent $\widehat{a}$ and $\widehat{N}$ can be 
explicitly connected to the time-dependent mass $m$ and frequency $\omega$. From (2.6) and (2.3), one can generally write

$$
\frac{d \delta}{d t}=\frac{\left(1+\delta^{2}\right)}{2 \sigma^{2}}-2 \omega^{2} \sigma^{2}, \quad \sigma=\sqrt{m\left(\frac{\sigma_{0}^{2}}{m_{0}}+\int_{t_{0}}^{t} \frac{\delta}{m} d t^{\prime}\right)}
$$

where $m_{0}=m\left(t_{0}\right)$ and $\sigma_{0}=\sigma\left(t_{0}\right)$. In principle, one might wonder about possible uses of (2.8) (equiv., (2.3)), to single out classes of nonautonomous quadratic systems having states with desired squeezing and mode production behaviors while evolving in time. It is evident that this might not be such a simple task, though. Let us argue in respect of this matter by focusing on cases yielding constant values for the position-momentum uncertainty relation. Provided that the function $\delta$ is constant, the Heisenberg uncertainty relations (2.7) are generally preserved under dynamical evolution. The case for vanishing covariance, hence minimum uncertainty, is clearly trivial and concerns the constant amplitude $\ell$ for the quasinormal mode regarding the related classical problem, say $\sigma=c \sqrt{m}$ (equiv., $2 c^{2} m \omega=1$ ), where $c$ is a constant [29]. In particular, when $m \omega=1$ (i.e., $c=1 / \sqrt{2}$ ), the eigenstates $|\alpha\rangle$ of $\widehat{a}$ are genuine coherent states, $\Delta_{\alpha} \widehat{q}=\Delta_{\alpha} \hat{p}=1 / \sqrt{2}$. The occurrence of constant, nonvanishing correlations, and hence of nonminimal stationary Heisenberg uncertainty relations (2.7), is instead obtained whenever mass and frequency are constrained by the relation

$$
m(t)=\frac{\omega_{0} m_{0}}{\omega(t)} e^{-2 \tilde{\delta}_{0} \int_{t_{0}}^{t} \omega\left(t^{\prime}\right) d t^{\prime}}, \quad \widetilde{\delta}_{0}=\frac{\delta_{0}}{\sqrt{1+\delta_{0}^{2}}}
$$

$\left(\delta=\delta_{0}=\right.$ const $\neq 0$; see $\left.(2.8)\right)$. In this case, $\sigma(t)=\sqrt[4]{\left(1+\delta_{0}^{2}\right)} / \sqrt{2 \omega}$. In other words, we have a class of time-dependent quadratic Hamiltonians whose simplest example is the celebrated Caldirola-Kanai Hamiltonian with a constant frequency and exponentially damped mass. This Hamiltonian probably represents the more natural from a physical perspective. However, it is interesting to note that the differential form of (2.9) is just a time-dependent Riccati-type equation, and that seeking a solution with constant mass (rather than constant frequency) produces a genuine parametric oscillator case, with $m=m_{0}$ and $\omega=(\text { const }+t)^{-1}$. There is in fact another way to look at the equation (2.9). The diffeomorphism $t \rightarrow$ $\omega^{(0)} \int_{t_{0}}^{t} \omega d t^{\prime}$ yields a time-dependent quadratic system with an effective constant frequency $\omega^{(0)}$, and an effective mass $(m \omega)^{\omega^{(0)}}$. There is no reason to be confined just to Kanai-Caldirola systems to observe constant Heisenberg uncertainty products. As one might expect on intuitive grounds as well as via elementary math, energy could flow into the system via the frequency parameter provided that there is a concomitant change in the mass parameter. The dynamical characteristics possibly shown by systems with a mass-frequency relation (2.9) are thereby merely inherited from those with constant mass damping, the major difference being in the difference in the passage of "laboratory" time $t$ and the systems "proper" time $\int_{t_{0}}^{t} \omega\left(t^{\prime}\right) d t^{\prime}$. 


\section{Features of the Eigenstates of $\widehat{I}$ Associated with Constant Correlation}

The description of the dynamics of quantum systems described by Hamiltonians (2.1) is implied in the adoption of time-dependent squeezing formulae. Indeed, the Schrödinger operators $\widehat{a}_{S, h o}, \widehat{a}_{S, h o}^{\dagger}$ realizing the instantaneous diagonalization of the Hamiltonian operator at the instant $t_{0}$ and the time-dependent operators $\widehat{a}, \hat{a}^{\dagger}$ can be generally mapped each into the other through a Bogolubov transformation [27, 29],

$$
\widehat{a}=\mu \widehat{a}_{S, h o}+v \widehat{a}_{S, h o}^{\dagger}, \quad|\mu|^{2}-|v|^{2}=1
$$

(among the others implying $\left.\Delta_{\alpha} \widehat{q} \Delta_{\alpha} \hat{p}=(1 / 2)|\mu+v||\mu-v|\right)$. In particular, if the evolution implied by (2.9) occurs, we easily find that the Bogolubov coefficients $\mu$ and $v$ vary according to

$$
\begin{aligned}
& \mu=\frac{1}{2}\left[\left(1-i \delta_{0}\right) Z+Z^{-1}\right], \quad v=\frac{1}{2}\left[\left(1-i \delta_{0}\right) Z-Z^{-1}\right], \\
& Z=\sqrt[4]{1+\delta_{0}^{2}} e^{\left(-\delta_{0} / \sqrt{1+\delta_{0}^{2}}\right) \int \omega} .
\end{aligned}
$$

In this context, the sought-after stationarity of the quantum correlations is, in practice, intimately connected to the assertion that the Hamiltonian and the invariant operators cannot have a common instantaneous spectral resolution at the instant when the "steady-behavior" starts to be observed. In fact, the coefficient $v$ above does not vanish at initial time $t_{0}$ because of the presence of an initial squeezing $\left(\delta, v \neq 0\right.$ at $\left.t_{0}\right)$ : at $t_{0}$, the ladder operators $\hat{a}$ and $\hat{a}^{\dagger}$ cannot transform into the ladder operators $\widehat{a}_{S, h o}$ and $\hat{a}_{S, h o}^{\dagger}$ of a standard harmonic oscillator with mass $m_{0}$ and frequency $\omega_{0}$. The eigenvectors resulting from the instantaneous diagonalization at time $t_{0}$ of the invariant $\widehat{I}$ cannot coincide with those of the Hamiltonian $\widehat{H}$ at the same time $\left(\langle n|\widehat{H}| n\rangle=(2 n+1) \sqrt{1+\delta_{0}^{2}} \omega\right)$.

Properties of the eigenvectors of the modal Fock basis $\{|n\rangle\}$ at arbitrary times are developed in terms of Wigner functions illustrated for low modes in Figure 1. There we see that states $|n\rangle$ exhibit squeezing, and less smooth transitions from classicality to nonclassicality than the corresponding eigenstates associated with the harmonic oscillator. As the Wigner functions evolve in phase space, these progressively spread out and diminish in magnitude, but converge about the center (as an effect of damping).

A complementary perspective is provided by the transition probabilities $P_{j \rightarrow k}=$ $\left|\langle j \mid k\rangle_{S, h o}\right|^{2}$ of states $|j\rangle$ with respect to the harmonic oscillator states $|k\rangle_{S, h o}$. Once the constant momenta ansatz (2.9) enters into consideration, these probabilities can generally be displayed as functions of the time-dependent frequency parameter $\omega(t)$, and of its time-integral $\int_{t_{0}}^{t} \omega\left(t^{\prime}\right) d t^{\prime}$, by adapting formulae derived in [31]. Straightforward inspection reveals their features, which can be essentially summarized as follows $((j, k) \neq(0,0))$ : (i) these are asymmetric bell-shaped curves, with a resulting decay rate slower than the initial increasing rate; (ii) as long as the constant value of momenta $\delta$ increases, the maximum is left-shifted (i.e., for a monotonically increasing frequency parameter $\omega$, the projection onto an initial-time Fock state is maximal much earlier in time with increasing $\delta$ ) and the suppression rate becomes 


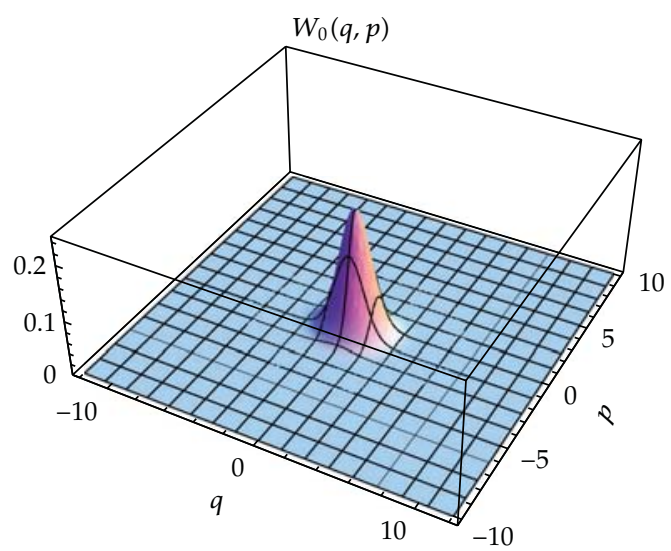

(a)

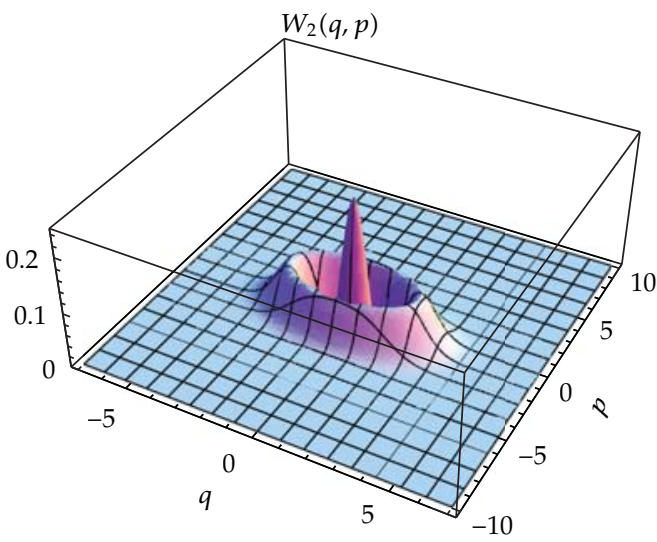

(c)

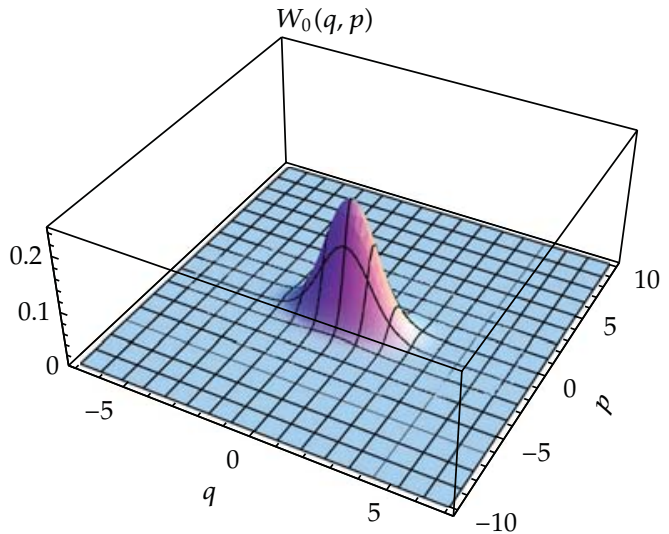

(b)

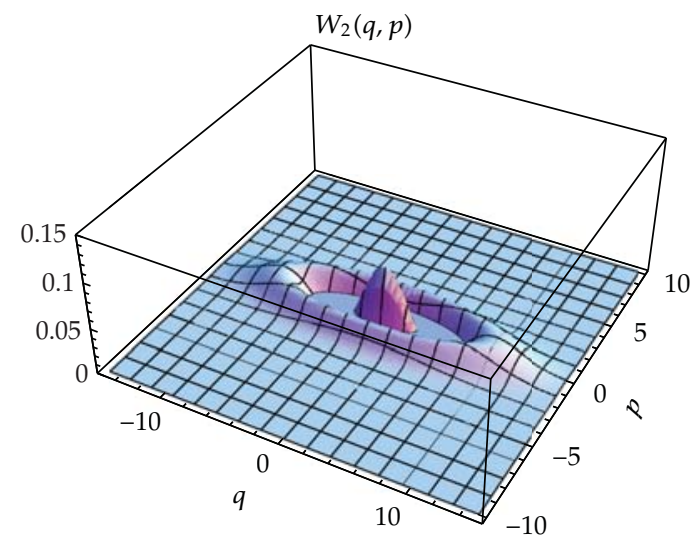

(d)

Figure 1: Wigner functions: (a) $|0\rangle$ for $\left(\omega, \int_{t_{0}}^{t} \omega\right)=(0.1,0)$; (b) $|0\rangle$ for $\left(\omega, \int_{t_{0}}^{t} \omega\right)=(0.1,0.005)$; (c) $|2\rangle$ for $\left(\omega, \int_{t_{0}}^{t} \omega\right)=(0.1,0.005) ;(\mathrm{d})|2\rangle$ for $\left(\omega, \int_{t_{0}}^{t} \omega\right)=(2.5,1.5) . \delta_{0}=1.7$ in all figures.

greater; and (iii) as functions of $\delta_{0}$, the $P_{n \rightarrow n}$ 's exhibit a pair of extremals, although as a kind of very marginal effect (see Figures 2 and 3 ).

Further insight is gained by looking at the second-order correlation functions

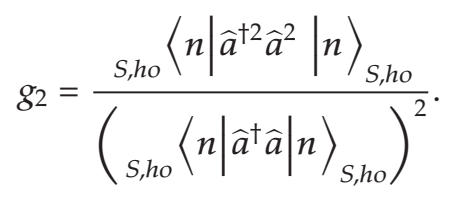

At the initial time, these assume the value

$$
g_{2}\left(t_{0}\right)=\frac{4\left(1+n+n^{2}\right)-4 \sqrt{1+\delta_{0}^{2}}(1+2 n)+3 \delta_{0}^{2}\left[\left(1+2 n+2 n^{2}\right)\right]}{\left[1+n-\sqrt{1+\delta_{0}^{2}}(1+3 n)\right]^{2}}
$$




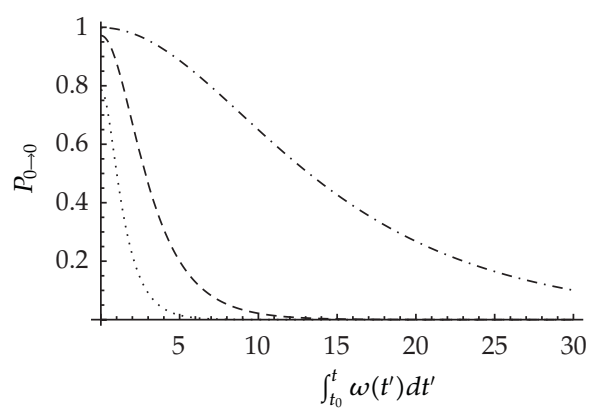

(a)

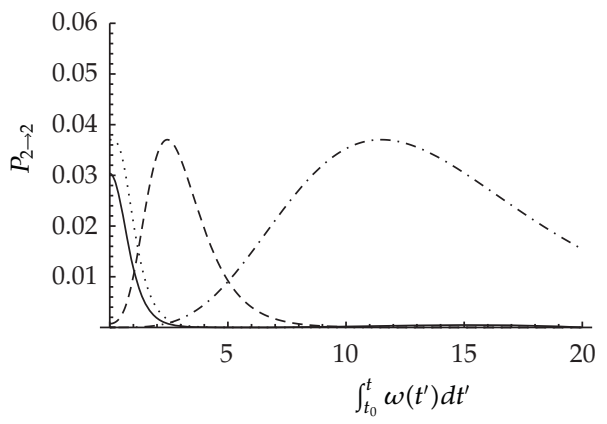

(c)

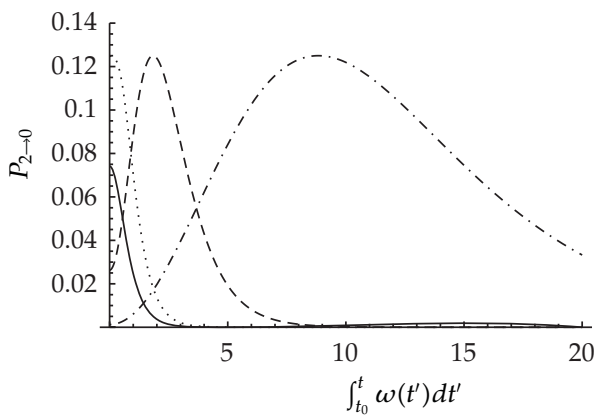

(b)

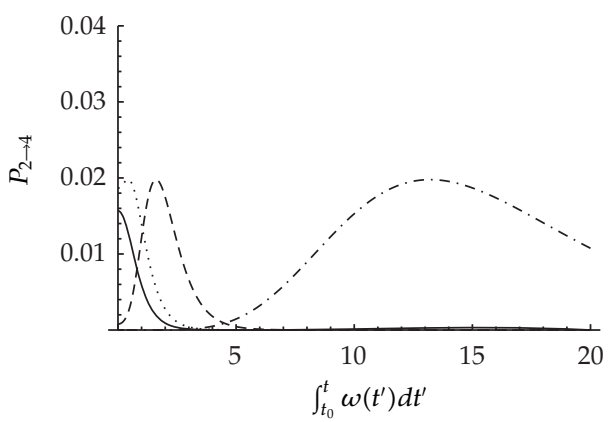

(d)

Figure 2: (a) $P_{0 \rightarrow 0}$ when $\delta_{0}=0.1,0.5,2$; (b) $P_{2 \rightarrow 0}$ when $\delta_{0}=0.1,0.5,3,10$; (c) $P_{2 \rightarrow 2}$ when $\delta_{0}=0.1,0.5,5,10$; (d) $P_{2 \rightarrow 4}$ when $\delta_{0}=0.1,1,5,15$. From lowest to highest values of $\delta_{0}$ : dot-dashed, dashed, dotted, solid.

which is always greater than the Poissonian value $(n-1) / n$ regardless of the specific values of $\delta_{0}$ and $n$ (which are monotonic increasing and functions), see Figure 4 . In the asymptotic limit $\int_{t_{0}}^{t} \omega\left(t^{\prime}\right) d t^{\prime} \rightarrow \infty, g_{2}\left(t_{0}\right)$ attains the limit value $3\left(1+2 n+2 n^{2}\right) /(1+2 n)^{2}$; in general, the greater the magnitude of $\delta_{0}$ is, the more rapidly the limit is approached.

\section{Wave Packets with Prescribed Steady Covariance Matrix Elements}

The picture obtained above for single eigenstates of the time-dependent annihilation operator $\widehat{a}$ and $\widehat{N}$ clearly survives on introducing the geometrical phases. Let us, therefore, characterize the stationary correlation features by considering solutions of the Schrödinger equation that are slightly more general than a single eigenstate $|n\rangle$ equipped with the LR phase. The geometrical phase dressing needed for the linear combination $|\Phi\rangle=\sum_{n} c_{n, 0}|n\rangle$ (the $c_{n, 0}$ 's being complex constants) of eigenstates $|n\rangle$ of $\widehat{N}$ that solves the Schrödinger equation is clearly obtained from the unitary operator

$$
\widehat{U}_{\varphi}=\sum_{n=0}^{\infty} e^{i \widehat{\varphi}_{j}}|n\rangle\langle n|, \quad \varphi_{j}=\partial_{t}^{-1}\left\langle j\left|i \partial_{t}-\widehat{H}\right| j\right\rangle
$$




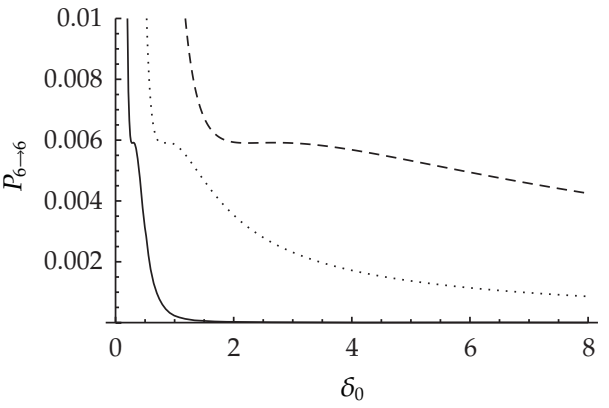

(a)

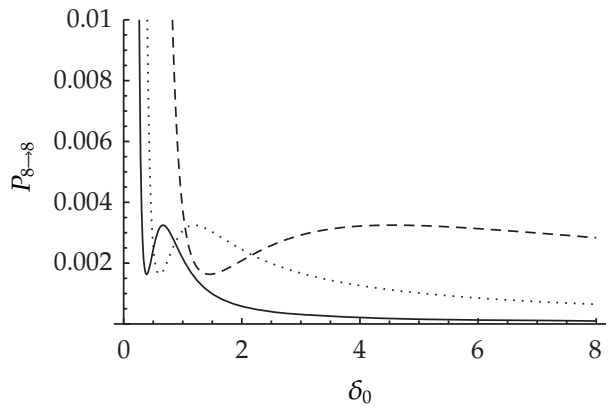

(b)

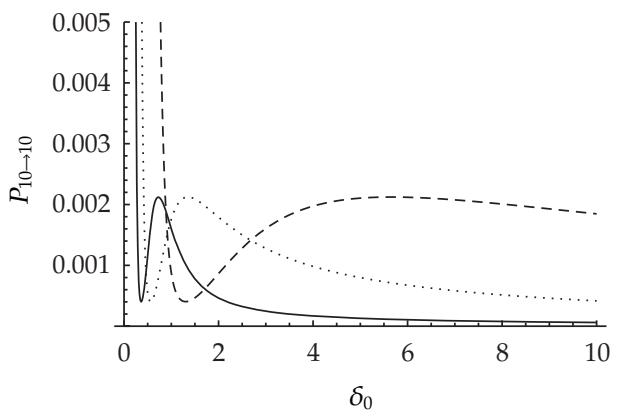

(c)

Figure 3: (a): $P_{6 \rightarrow 6}$ versus $\delta_{0}$ for $\int_{t_{0}}^{t} \omega\left(t^{\prime}\right) d t^{\prime}=1,2,5$ (solid, dashed, dotted); (b)-(c) $P_{8 \rightarrow 8}$ and $P_{10 \rightarrow 10}$ versus $\delta_{0}$ versus $\delta_{0}$ for $\int_{t_{0}}^{t} \omega\left(t^{\prime}\right) d t^{\prime}=1,2,3$ (dashed, dotted, solid).

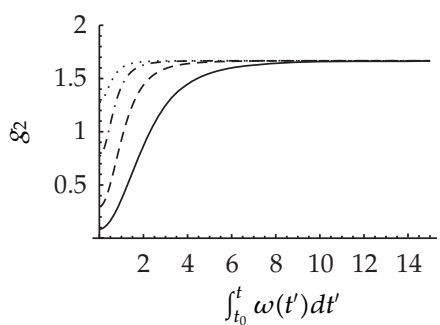

(a)

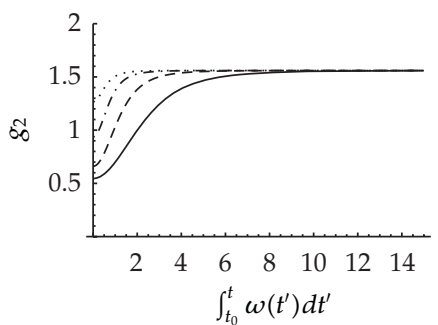

(b)

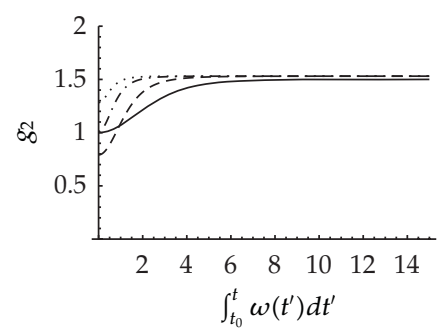

(c)

Figure 4: Second-order correlation functions evaluated over (a) the $n=1$, (b) the $n=2$, and (c) the $n=50$ harmonic oscillator eigenstate that results from the instantaneous Hamiltonian spectral problem at $t_{0}$. In all figures: $\delta_{0}=0.2,0.4,0.8,1.6$ (solid, dashed, dot-dashed, dotted).

That is, one has to consider the superpositions [18]

$$
|\psi\rangle=\widehat{U}_{\varphi}|\Phi\rangle=\sum_{n} c_{n, 0} e^{i \varphi_{n}(t)}|n\rangle, \quad \varphi_{j}=-\left(j+\frac{1}{2}\right) \int_{t_{0}}^{t} \frac{d t^{\prime}}{\sigma^{2}}
$$




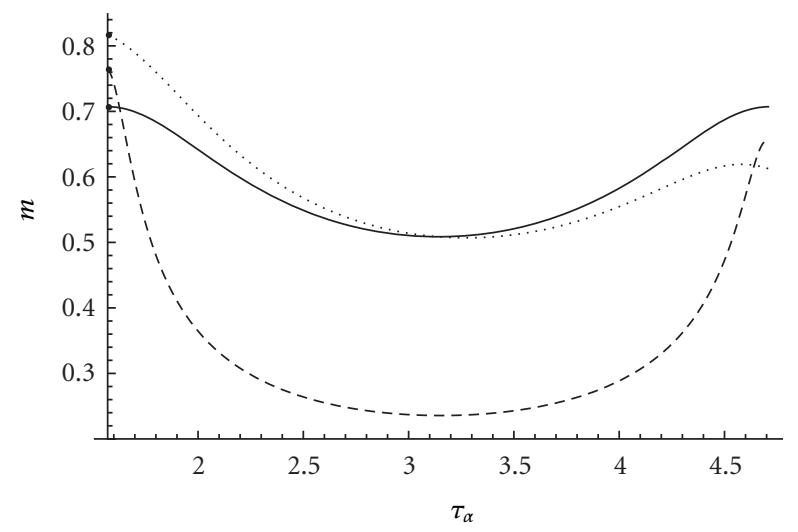

Figure 5: Function $m$ of $(4.15)$ in the interval $(\pi / 2,3 \pi / 2)$ when $\left(|\alpha|^{2}, \Delta^{(0)}\right)=(0.5,0)$ (solid), $\left(|\alpha|^{2}, \Delta^{(0)}\right)=$ $(0.5,0.3)$ (dotted), and $\left(|\alpha|^{2}, \Delta^{(0)}\right)=(20,0.9)$ (dashed); both $\sigma_{c, 0}$ and the right-hand side of $(4.15)$ have been set to the unity.

The entries of the position-momentum correlation matrix associated with the wave-packet (4.2) are identified through

$$
\left(\Delta_{\psi} \widehat{q}\right)^{2}=\frac{\sigma^{2}}{m} a, \quad\left(\Delta_{\psi} \widehat{p}\right)^{2}=\frac{m}{4 \sigma^{2}}\left(b+c \delta+a \delta^{2}\right), \quad \Delta_{\psi}(\widehat{q}, \widehat{p})=2 c+a \delta
$$

where the time-dependent coefficients $a, b, c$ assume obvious standard forms that depend on normalization constants and sines and cosines of phase differences. Descriptions of interference patterns that arise from the related geometrical phases are obtained by evaluating differences between the phases $\phi_{r}$ of (4.2). As Lewis-Riesenfelds total geometric phase $\varphi_{j}$ that can be associated with each eigenstate $|j\rangle$ depends linearly on $j$, then, for whatever the specific linear combination one has, the time-dependence of the interferences is basically governed by just the single-phase $\varphi_{0}$. Despite this, understanding how the desired properties of those quantities in (4.3) might be obtained is still not obvious: it generally requires solving highly nonlinear differential equations that couple both mass and frequency time-dependent parameters.

Let us focus on how one derives the oscillator parameters, as well as select and weight the states of wave packets, to produce a prescribed constant value $\Delta^{(0)}$ for the position-momentum correlation $\Delta_{\psi}(\widehat{q}, \hat{p})$ on $|\psi\rangle$. The lesson we learned from the previous section is that precise relationships between mass and frequency are needed to generate Fock- or coherent-type states on which squeezing characteristics can be imposed ab initio and that these are sufficiently robust in respect to correlations. Naively, we might say that the way the Hamiltonian kinetic energy term and the energy pumped into the system through the potential varies during evolution is such that if the states are initially properly chosen there is an effective dynamical balance of their roles at successive times. The remark we make here is that more general structures of the type (4.2) for which the correlations are robust with respect to the dynamics can be identified as well if mass and frequency parameters are properly connected one to the other via other relationships. The possibility of identifying non-empty sets of states enjoying the property jointly with the associated parameter conditions, by avoiding highly complicated nonlinear differential equations while 


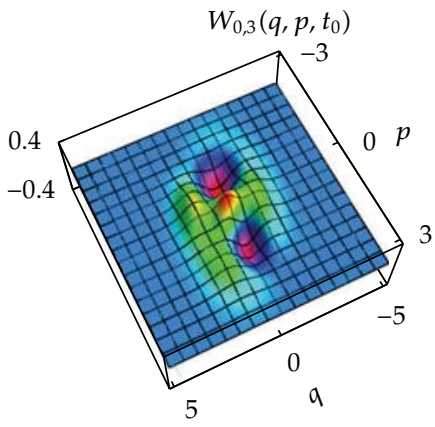

(a)

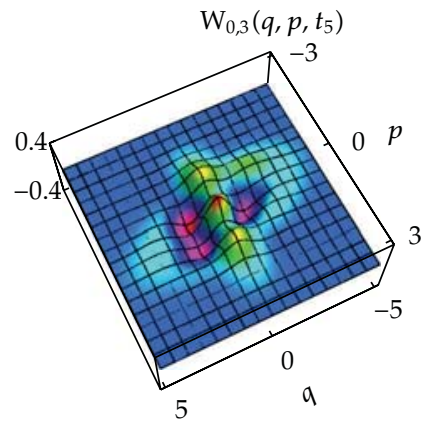

(d)

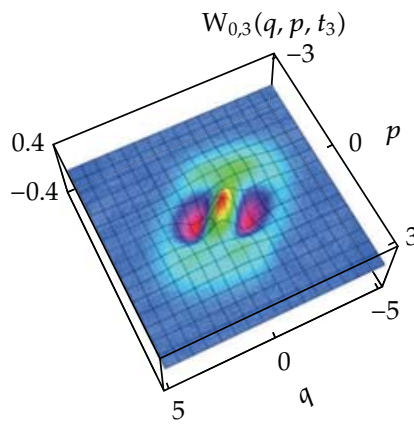

(b)

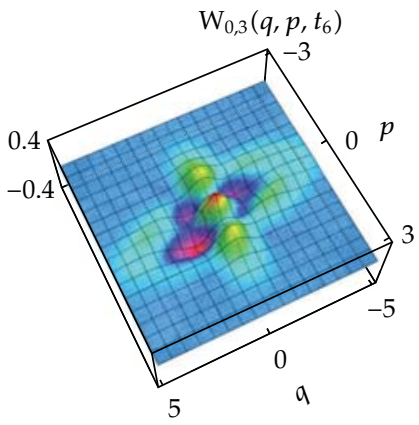

(e)

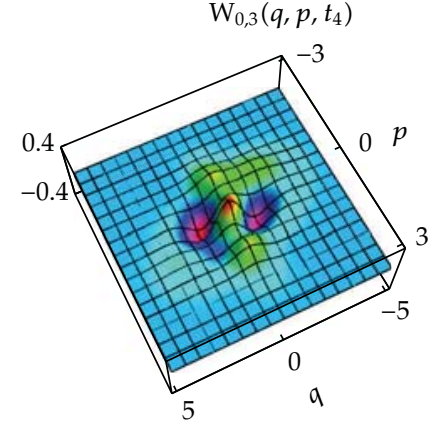

(c)

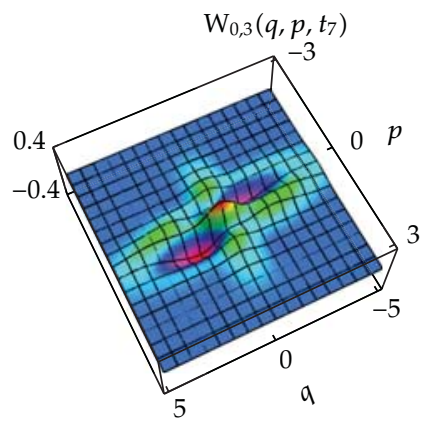

(f)

Figure 6: Wigner functions of $(1 / \sqrt{2})(|0\rangle+|3\rangle)$ at successive times; $\delta_{0}=0.4$.

constraining the topological phases $\varphi_{j}$, is very simply supported within the very basic mathematical framework. One needs to focus on the simplest choice for the function $\sigma$. It is concerned with constant $\sigma^{\prime}$ s, so that it is the mass parameter only that characterizes the time dependence of the right hand side of identities in (4.3). In turn, this means that the Ermakov equation (2.3) is in fact equivalent to a condition for just the frequency parameter:

$$
\omega^{2}=\frac{1}{2} \frac{d M}{d t}+\frac{M^{2}}{4}+\frac{1}{\sigma_{c}^{4}}
$$

where $\sigma_{c}$ is a constant, whereas $M=(1 / m)(d / d t) m$ follows from the condition one wishes to prescribe. In this manner, mass and frequency parameters are obtained. A constant value for the Heisenberg variances product can then be attained straightforwardly, which we illustrate by means of examples in terms of two Fock eigenstates of the invariant operator $\widehat{N}$,

$$
\left|\Psi_{(k, s)}\right\rangle=c_{k, 0} e^{\varphi_{k}}|k\rangle+c_{s, 0} e^{i \varphi_{s}}|s\rangle
$$

If $s \geq k+3$, with the constant and normalized $c_{j, 0}=\left|c_{j, 0}\right| e^{\phi_{j}{ }^{\prime}}$, it is trivially seen that the constancy of the covariance (as well as of Heisenberg uncertainty) is found if $\delta$ is constant. As confirmed by Wigner functions in Figures 6, 7, 8 and 9 a general mechanism occurs that tends to symmetrize the shape of Wigner functions in phase space and to single out different peaks exhibiting squeezing dynamics similar to that characterizing Fock eigenstates $|k\rangle$. A rotation 


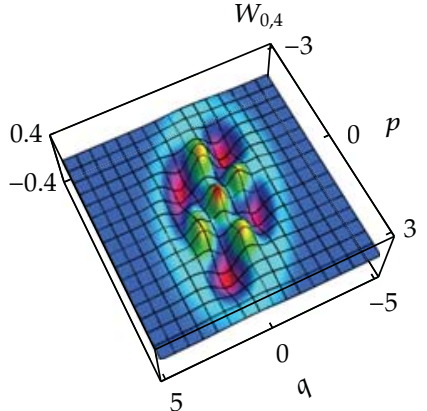

(a)

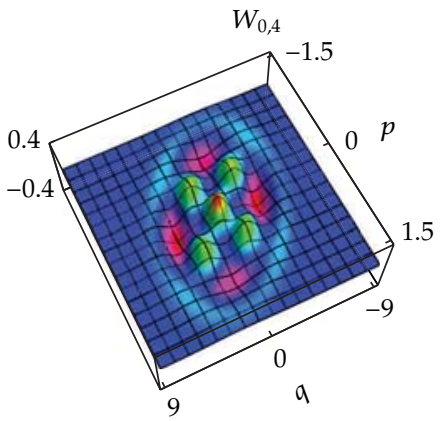

(d)

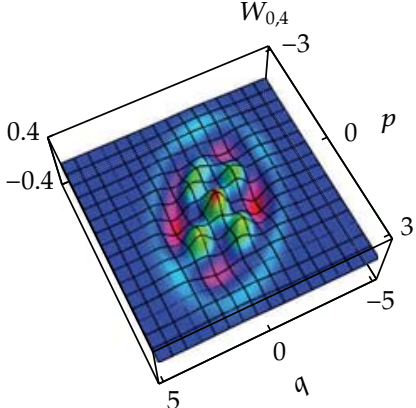

(b)

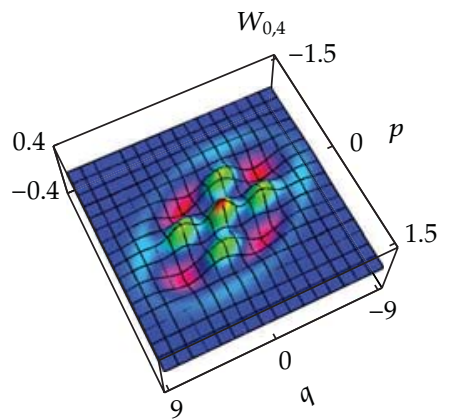

(e)

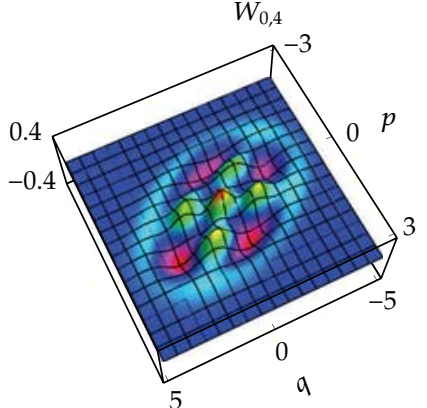

(c)

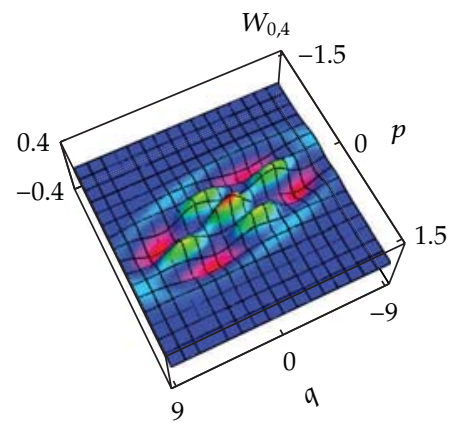

(f)

Figure 7: Wigner functions of $(1 / \sqrt{2})(|0\rangle+|4\rangle)$ at successive times; $\delta_{0}=0.4$.

intervenes in phase space as a consequence of squeezing of the momentum variance, along with weak fluctuation of the background level outside the regions where the transitions from classicality to nonclassicality (and vice versa) have a major effect. These are general features. However, if quantum numbers of modal eigenstates entering into the linear combination (4.5) are too close (i.e., $s=k+1, k+2$ ), then initial interference terms affect the properties of the Wigner function too much, and the initial values for correlations are not robust with respect to time evolution. For instance, querying constant covariance over $\left|\Psi_{(k, k+2)}\right\rangle$ would be equivalent to having dynamics such that

$$
\frac{\sigma^{2}}{m} \sqrt{\Gamma_{0}+\Gamma_{1} \cos \tau} e^{\left(-\Delta^{(0)} / \sqrt{\Gamma_{0}^{2}-\Gamma_{1}^{2}}\right) \tan ^{-1}\left(\left(\sqrt{\Gamma_{0}-\Gamma_{1}} / \sqrt{\Gamma_{0}+\Gamma_{1}}\right) \tan (\tau / 2)\right)}=\text { const, }
$$

with $\tau=2 \int\left(d t^{\prime} / \sigma^{2}\right)+\left(\phi_{k}-\phi_{k+2}\right)$ and

$$
\Gamma_{0}=\left(1+2 k+4\left|c_{k+2,0}\right|^{2}\right), \quad \Gamma_{1}=2 \frac{\left|c_{k+2,0}\right| \sqrt{1-\left|c_{k+2,0}\right|^{2}}}{\sqrt{(k+1)(k+2)}}
$$

Clearly, the case must be treated with care because of the tan-term. A natural time window enters into the description of the system, which is basically defined by the range allowed for $\tau,(-\pi / 2, \pi / 2)(\bmod (\pi))$. A nonvanishing covariance would carry a discontinuous term into the mass/frequency correspondence. If one is willing to afford the description 


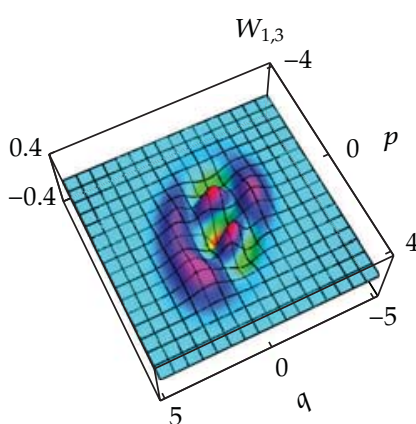

(a)

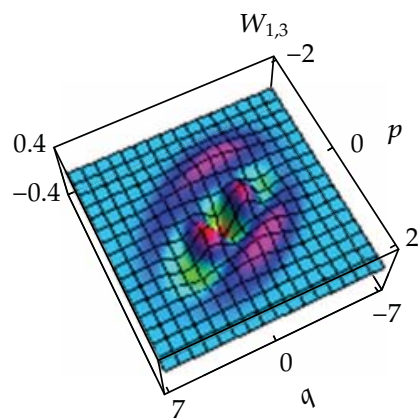

(d)

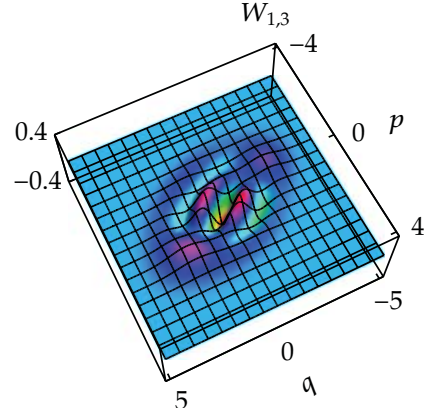

(b)

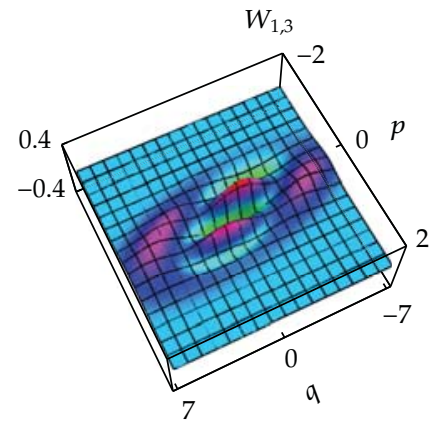

(e)

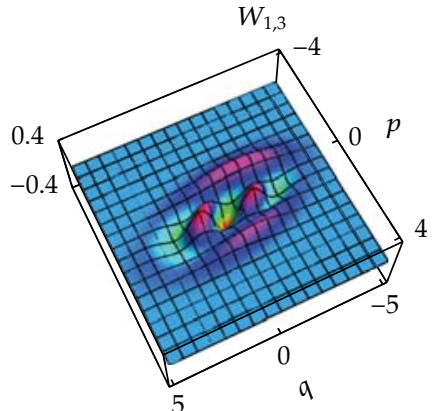

(c)

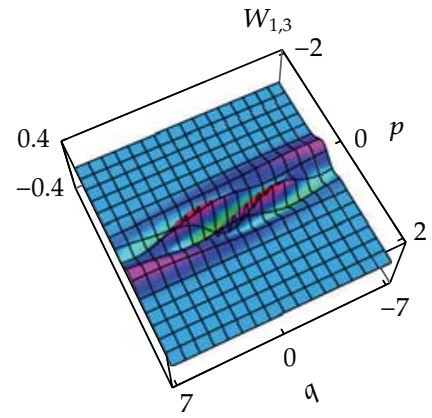

(f)

Figure 8: Wigner functions of $(1 / \sqrt{2})(|1\rangle+|3\rangle)$ at successive times; $\delta_{0}=0.4$.

of dynamics in the case of noncontinuous parameters, then one always has to remember that whenever a discontinuity occurs in $\sigma$ and $m$ then a change of Fock basis and a matching of the Bogolubov coefficients is generally required. Requiring that covariance should always vanish would provide instead protection from such discontinuities. If both the ansatz $\sigma=\sigma_{c, 0}=$ const and $\Delta^{(0)}=0$ were to be considered, then

$$
m^{2} \alpha \Gamma_{0}+\Gamma_{1} \cos F(t), \quad F(t)=\frac{t_{0}-t}{2 \sigma_{c, 0}^{2}}+\phi_{k+2}-\phi_{k}
$$

In turn, the mass / frequency correspondence of (4.4) would finally yield

$$
\sigma_{c, 0}^{4} \omega^{2}=1+\left[\frac{\Gamma_{1} \sin F}{2\left(\Gamma_{0}+\Gamma_{1} \cos F\right)}\right]^{2}
$$

For wave-function $\left|\Psi_{(k, k+1)}\right\rangle$, one expects a slight difficulty as the presence of the term

$$
\tan \left[\int^{t} \frac{d t^{\prime}}{\sigma^{2}}+\left(\phi_{k}-\phi_{k+1}\right)\right]
$$




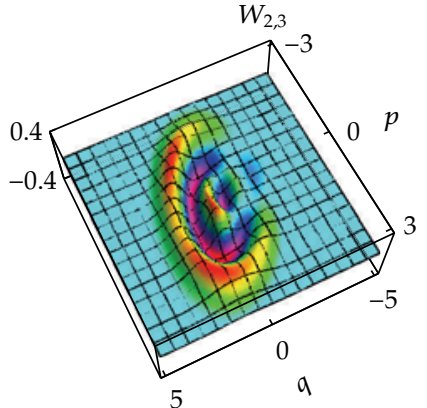

(a)

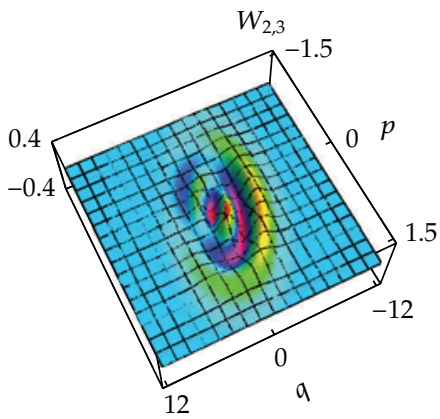

(d)

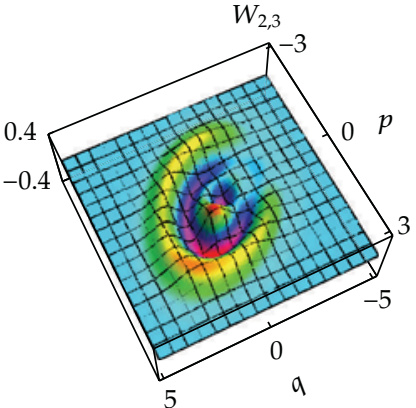

(b)

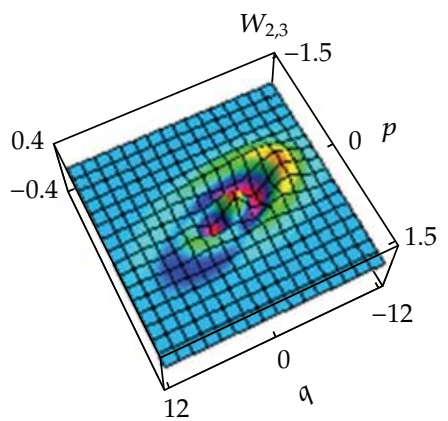

(e)

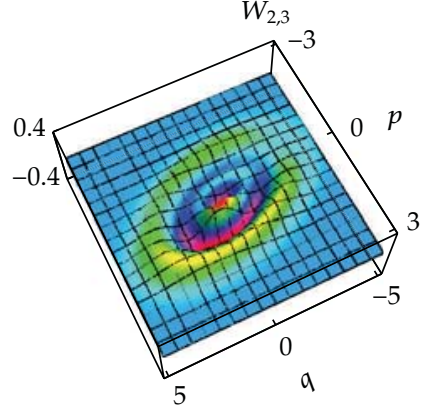

(c)

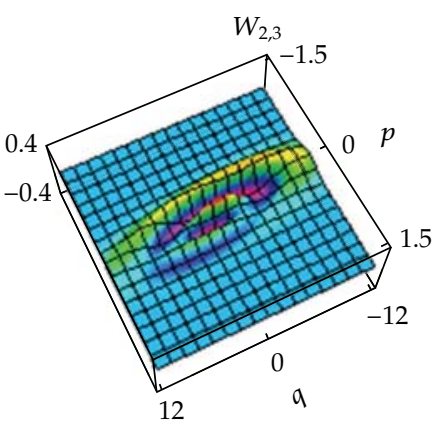

(f)

Figure 9: Wigner functions of $(1 / \sqrt{2})(|2\rangle+|3\rangle)$ at $t_{0}$ and at successive times; $\delta_{0}=0.4$.

implies that the reality of the formal condition for the function $\ell$ features only if

$$
2\left|c_{k+1}\right|^{2}\left(1-\left|c_{k+1}\right|^{2}\right)\left\{1+\cos \left[2 \int^{t} \frac{d t^{\prime}}{\sigma^{2}}+2\left(\phi_{k}-\phi_{k+1}\right)\right]\right\}>(k+1)\left(1+2 k+2\left|c_{k+1}\right|^{2}\right) .
$$

This is possible only if $k=0$ and $\left|c_{k+1,0}\right|$ is less than $(5-\sqrt{13}) / 4$ (the lesser this is, the wider is the range of the cosine argument).

\subsection{Lewis-Riesenfeld Coherent and Cat States}

Before discussing states expanded as linear combinations of eigenvectors $|\alpha\rangle$ corresponding to the time-dependent annihilation operator $\widehat{a}$, it is worth noting that they are no solutions to the time-dependent Schrödinger equation. A more useful set of states than the LewisRiesenfeld basis would be one constructed by letting the unitary operator,which accounts for the geometric phase dressing act on each of its elements associated with a constant eigenvalue $\alpha,\left\{|\alpha\rangle_{\varphi}=\widehat{U}_{\varphi}|\alpha\rangle\right\}$. Since $|\alpha\rangle_{\varphi}=e^{i \varphi_{0}}\left|e^{i 2 \varphi_{0}} \alpha\right\rangle$, states are, therefore, still eigenstates of the timedependent annihilation operator, but with complex eigenvalues, which are phase-shifted by twice the vacuum geometrical phase with respect to the eigenvalue of the "naked" LR state $|\alpha\rangle$. Most importantly, for any constant $\alpha$, the vector state $|\alpha\rangle_{\varphi}=\widehat{U}_{\varphi}|\alpha\rangle$ is just a solution to the time-dependent Schrödinger equation (with initial squeezed coherent form fixed by the relationship between the instantaneous diagonalization at $t_{0}$ of the Hamiltonian (2.1) and 
the invariant (2.2), that is, by the Bogolubov relations (2.5) and (3.1) at $t_{0}$ ). The remark is as naturally crucial in applications having as necessary ingredients combinations of the $|\alpha\rangle$ 's. For instance, for standard Schrödinger cat states, this means that an implementation of their definition according to

$$
|\tilde{\Psi}\rangle=\widetilde{\mathcal{N}}_{\mathrm{cat}}\left(|\alpha\rangle_{\varphi}+|-\alpha\rangle_{\varphi}\right), \quad \widetilde{\mathcal{N}}_{\mathrm{cat}}=\left(2+e^{-2|\alpha|^{2}}\right)^{-1 / 2}
$$

( $\alpha=$ const) would be in order. The interesting probabilities can be easily computed; in particular,

$$
\begin{aligned}
& \left\langle\tilde{\Psi}\left|\widehat{p}^{2}\right| \tilde{\Psi}\right\rangle=\widetilde{\mathcal{N}}_{\text {cat }}^{2} \frac{m}{\sigma^{2}}\left\{\left(1+\delta^{2}\right)+4 \operatorname{Re}^{2}\left[(\delta-i) \alpha e^{i 2 \varphi_{0}}\right]\right\} \\
& +{\widetilde{\mathcal{N}_{\text {cat }}}}^{2} \frac{m}{\sigma^{2}} e^{-2|\alpha|^{2}}\left\{\left(1+\delta^{2}\right)-4 \operatorname{Im}^{2}\left[(\delta-i) \alpha e^{i 2 \varphi_{0}}\right]\right\}, \\
& \langle\widetilde{\Psi}|\widehat{q} \hat{p}+\widehat{p} \widehat{q}| \widetilde{\Psi}\rangle=8{\widetilde{\mathcal{N}_{\text {cat }}}}^{2} \operatorname{Re}\left(\alpha e^{i 2 \varphi_{0}}\right) \operatorname{Im}\left(\alpha e^{i 2 \varphi_{0}}\right)\left(1+e^{-2|\alpha|^{2}}\right) \\
& +2 \widetilde{\mathcal{N}_{\mathrm{cat}}^{2}} \delta\left\{\left[1+4 \operatorname{Re}^{2}\left(\alpha e^{i 2 \varphi_{0}}\right)\right]+\delta e^{-2|\alpha|^{2}}\left[1-4 \operatorname{Im}^{2}\left(\alpha e^{i 2 \varphi_{0}}\right)\right]\right\} .
\end{aligned}
$$

A constant covariance $\Delta^{(0)}$ would be thus attained for $|\tilde{\Psi}\rangle$ provided that

$$
\begin{gathered}
\frac{\sigma^{2}}{m} \frac{\exp \left\{\left(-\Delta^{(0)}\left(2+e^{-2|\alpha|^{2}}\right) / \sqrt{\beta_{1}} \sqrt{\beta_{1}+\beta_{2}}\right) \tan ^{-1}\left[\left(\sqrt{\beta_{1}} / \sqrt{\beta_{1}+\beta_{2}}\right) \tan \left(\varphi_{\alpha}-2 \varphi_{0}\right)\right]\right\}}{\sqrt{2 \beta_{1}+\beta_{2}\left[1+\cos 2\left(\varphi_{\alpha}-2 \varphi_{0}\right)\right]}}=\text { const, } \\
\beta_{1}=\left(1-4|\alpha|^{2}\right) e^{-2|\alpha|^{2}}, \quad \beta_{2}=8|\alpha|^{2}\left(1+e^{-2|\alpha|^{2}}\right),
\end{gathered}
$$

is constant $\left(\alpha=|\alpha| e^{i \varphi_{\alpha}}\right)$. Distinct working hypotheses can be considered to analyze the allowed cases. But since $\tau_{\alpha}=\varphi_{\alpha}-2 \varphi_{0}$ is assumed to vary, the discontinuity of mass parameters during evolution is needed to sustain a nonvanishing constant covariance. That implies the possible breaking of the mass parameter curve associated with vanishing covariance (Figure 5). The steadiness condition cannot survive the loss of coherence mechanism imposed by dynamics beyond the characteristic proper time window, unless it is sustained by a suitable instantaneous jump of the first derivative of the mass parameter to restore a likely initial configuration (after one typical period, the same arguments do evidently apply again). In particular, the choice of the constancy of the auxiliary function $\sigma$ immediately turns into the condition that the mass (and consequently the frequency through (4.4)) varies according to a modified exponential law in defined time intervals (Figure 5).

\section{Discussion}

We considered position-momentum covariance matrix elements associated with solutions of the Schrödinger equation for systems for which the dynamics is ruled by a quadratic Hamiltonian with time-dependent mass and frequency parameters. In particular, we paid attention to 
the possibility that states with prescribed constant correlations can be constructed. It turns out that correlations can be reasonably robust during dynamics if energy appropriately flows into the system. At first, one might erroneously believe that the constancy of correlation matrix elements represents a kind of mild effect reflecting the circumstance that an adiabatic (or pretty close to it) regime is necessarily taking place. This conclusion is not correct, in general. The stationarity of the position-momentum Heisenberg uncertainty relation (2.7) is shown provided that (2.9) occurs, in fact. As can easily be inferred by introducing parameters related to adiabaticity $\varepsilon$ and slow-varying time $\tau$ as customary, for all quadratic Hamiltonians, the mass-frequency relationship associated with such evolutions reads $m \omega=$ const, neglecting $\dot{M}^{2}$ and $M^{2}$ and the search for likely frequency dependence. Significantly, this implies $\ell=$ const and the minimality of the Heisenberg uncertainty among LR states $|\alpha\rangle$. The dynamical condition for constant covariance means instead that $m \omega \ell^{2}=$ const. The limit $\ell \propto e^{2 \tilde{\delta}_{0} \int \omega d t^{\prime}} \rightarrow$ $\ell=$ const, therefore, promotes the stationarity nonminimality condition (2.9) over states $|n\rangle$ (likewise $|\alpha\rangle$ ) to that of null covariance. Also, bearing in mind that in the adiabatic regime the geometric phase term merely reduces to an integral over the time-dependent frequency parameter [32], the function $\delta$ characterizing the sought dynamical regime is completely absent. The rate of the exponential term in the right hand side of (2.9), therefore, quantifies the needed deviation from adiabaticity of the processes thereby preserving the steadiness of covariances among modal Fock states $|n\rangle$ and Lewis-Riesenfeld generalized coherent states. Details have been, therefore, provided in regard to features displayed by quantum states associated with nonautonomous quadratic Hamiltonian within the dynamics implied by stationarity of the Heisenberg uncertainty and/or correlations. Once the analysis is performed within the invariant framework and by exploiting the Bogolubov mappings, the case is very naturally introduced. In its simplest form, it can be formally treated with elementary algebraic math. Nevertheless, its analysis is significant in that it provides arguments to support counterintuitive examples of wave-packets for nonautonomous systems that do not change position-momentum correlation properties during evolution in spite of the fact that these (i.e., the shapes) actually decohere. A key point remains in identifying proper energy inflows and suitable initially squeezed states that exhibit the property of maintaining of the steadiness of correlations, at least for some time intervals (see (4.6) and (4.15)).

\section{References}

[1] R. K. Colegrave and M. Sebawe Abdalla, "Harmonic oscillator with exponentially decaying mass," Journal of Physics A, vol. 14, no. 9, pp. 2269-2280, 1981.

[2] C. K. Law, "Effective Hamiltonian for the radiation in a cavity with a moving mirror and a timevarying dielectric medium," Physical Review A, vol. 49, no. 1, pp. 433-437, 1994.

[3] I. A. Pedrosa, "Quantum electromagnetic waves in nonstationary linear media," Physical Review A, vol. 83, no. 3, Article ID 032108, 2011.

[4] I. A. Pedrosa, C. Furtado, and A. Rosas, "Light propagation: from dielectrics to curved spacetimes," EPL, vol. 94, no. 3, Article ID 30002, 2011.

[5] D. F. Walls and G. J. Milburn, Quantum optics, Springer-Verlag, Berlin, Second edition, 2008.

[6] N. C. Menicucci and G. J. Milburn, "Single trapped ion as a time-dependent harmonic oscillator," Physical Review A, vol. 76, no. 5, Article ID 052105, 2007.

[7] L. P. Grishchuk and Y. V. Sidorov, "Squeezed quantum states of relic gravitons and primordial density fluctuations," Physical Review D, vol. 42, no. 10, pp. 3413-3421, 1990.

[8] A. Geralico, G. Landolfi, G. Ruggeri, and G. Soliani, "Novel approach to the study of quantum effects in the early universe," Physical Review D, vol. 69, no. 4, Article ID 043504, 2004.

[9] G. Landolfi and G. Soliani, "Trans-Planckian effects in nonlinear-dispersion cosmologies," Physics Letters B, vol. 588, no. 1-2, pp. 1-6, 2004. 
[10] A. M. M. De Carvalho, C. Furtado, and I. A. Pedrosa, "Scalar fields and exact invariants in a Friedmann-Robertson-Walker spacetime," Physical Review D, vol. 70, no. 12, Article ID 123523, 2004.

[11] I. A. Pedrosa, C. Furtado, and A. Rosas, "Exact linear invariants and quantum effects in the early universe," Physics Letters B, vol. 651, no. 5-6, pp. 384-387, 2007.

[12] C. E. F. Lopes, I. A. Pedrosa, C. Furtado, and A. M. d. M. Carvalho, "Gaussian wave packet states of scalar fields in a universe of de Sitter," Journal of Mathematical Physics, vol. 50, no. 8, Article ID 083511, 2009.

[13] K. Bakke, I. A. Pedrosa, and C. Furtado, "Geometric phases and squeezed quantum states of relic gravitons," Journal of Mathematical Physics, vol. 50, no. 11, Article ID 113521, 2009.

[14] I. A. Pedrosa, K. Bakke, and C. Furtado, "Gaussian wave packet states of relic gravitons," Physics Letters B, vol. 671, no. 2, pp. 314-317, 2009.

[15] H. Qin and R. C. Davidson, "Symmetries and invariants of the oscillator and envelope equations with time-dependent frequency," Physical Review Special Topics-Accelerators and Beams, vol. 9, no. 5, Article ID 054001, 2006.

[16] J. Struckmeier, "Hamiltonian dynamics on the symplectic extended phase space for autonomous and non-autonomous systems," Journal of Physics A, vol. 38, no. 6, pp. 1257-1278, 2005.

[17] J. Struckmeier and C. Riedel, "Canonical transformations and exact invariants for time-dependent Hamiltonian systems," Annalen der Physik, vol. 11, no. 1, pp. 15-38, 2002.

[18] H. R. Lewis and W. B. Riesenfeld, "An exact quantum theory of the time-dependent harmonic oscillator and of a charged particle in a time-dependent electromagnetic field," Journal of Mathematical Physics, vol. 10, no. 8, pp. 1458-1473, 1969.

[19] V. V. Dodonov and V. I. Man'Ko, "Coherent states and the resonance of a quantum damped oscillator," Physical Review A, vol. 20, no. 2, pp. 550-560, 1979.

[20] C.-I. Um, K.-H. Yeon, and T. F. George, "The quantum damped harmonic oscillator," Physics Reports, vol. 362, no. 2-3, pp. 63-192, 2002.

[21] G. Landolfi, "Weyl-ordered series form for the angle variable of the time-dependent oscillator," Journal of Physics A, vol. 41, no. 18, Article ID 185302, 2008.

[22] V. V. Dodonov and V. I. Man'ko, "Invariants and the Evolution of Non-stationary Quantum Systems," in Proceedings of Lebedev Physics Institute, M. A. Markov, Ed., vol. 183, Nova Science, Commack, NY, USA, 1989.

[23] F. Haas, "The damped Pinney equation and its applications to dissipative quantum mechanics," Physica Scripta, vol. 81, no. 2, Article ID 025004, 2010.

[24] M. Razavy, Classical and Quantum Dissipative Systems, Imperial College Press, London, UK, 2005.

[25] E. Kanai, "On the quantization of the dissipative systems," Progress of Theoretical Physics, vol. 3, no. 4, pp. 440-442, 1948.

[26] P. Caldirola, "Quantum theory of nonconservative systems," Nuovo Cimento. B. Serie 11, vol. 77, no. 2, pp. 241-262, 1983.

[27] G. Profilo and G. Soliana, "Group-theoretical approach to the classical and quantum oscillator with time-dependent mass and frequency," Physical Review A, vol. 44, no. 3, pp. 2057-2065, 1991.

[28] S. K. Suslov, "Dynamical invariants for variable quadratic Hamiltonians," Physica Scripta, vol. 81, no. 5, Article ID 055006, 2010.

[29] G. Landolfi, G. Ruggeri, and G. Soliani, "A note on the loss of coherence in wave packets squeezed systems," International Journal of Quantum Information, vol. 2, no. 4, pp. 529-540, 2004.

[30] I. A. Pedrosa, "Comment on Coherent states for the time-dependent harmonic oscillator," Physical Review D, vol. 36, no. 4, pp. 1279-1280, 1987.

[31] H. P. Yuen, "Two-photon coherent states of the radiation field," Physical Review A, vol. 13, no. 6, pp. 2226-2243, 1976.

[32] D. A. Morales, "Correspondence between Berry's phase and Lewis's phase for quadratic Hamiltonians," Journal of Physics A, vol. 21, no. 18, pp. L889-L892, 1988. 


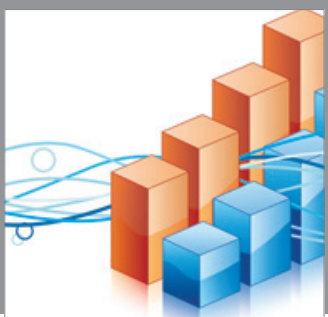

Advances in

Operations Research

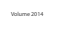

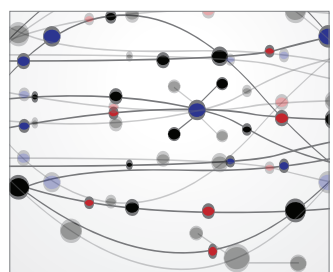

\section{The Scientific} World Journal
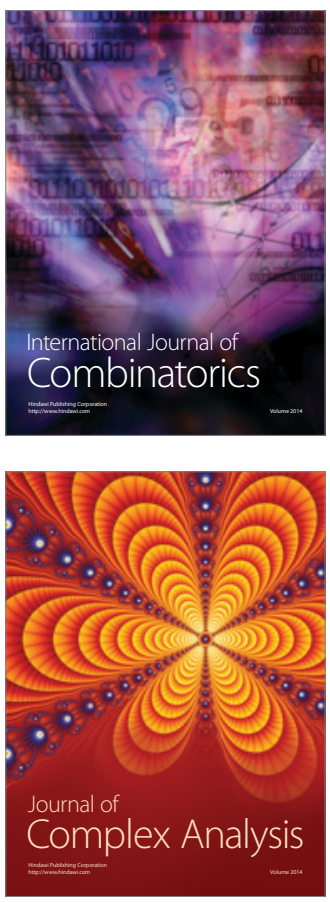

International Journal of

Mathematics and

Mathematical

Sciences
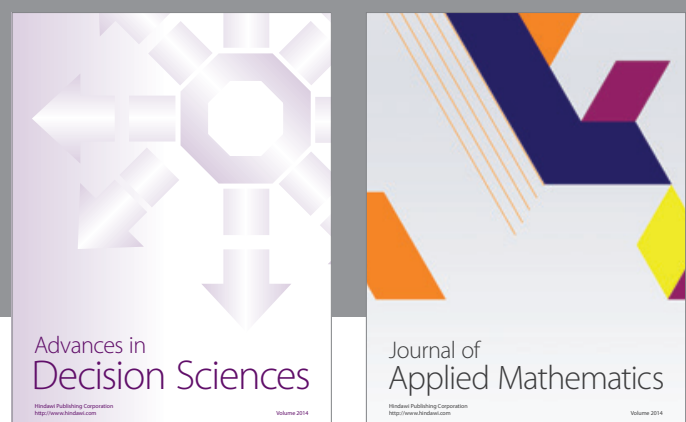

Journal of

Applied Mathematics
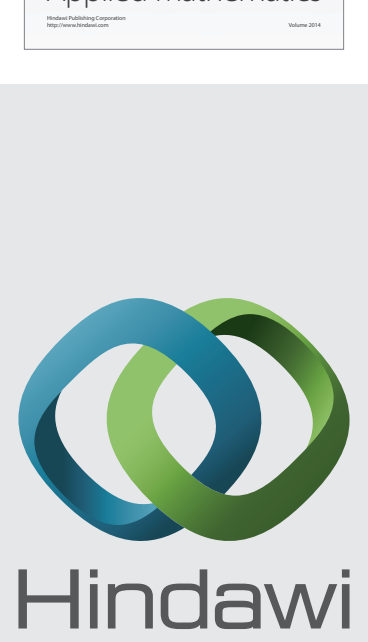

Submit your manuscripts at http://www.hindawi.com
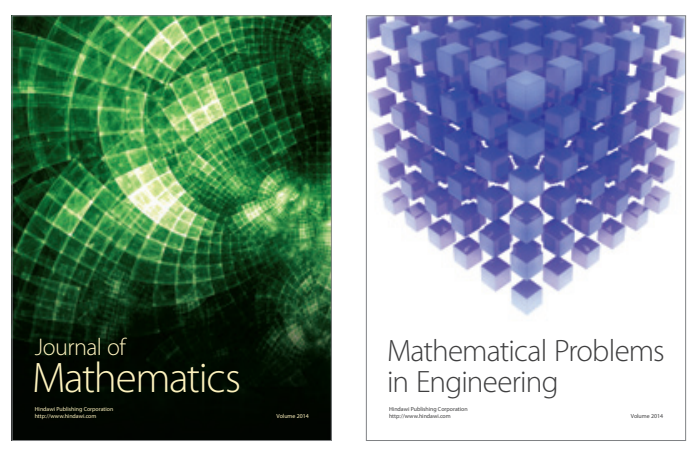

Mathematical Problems in Engineering
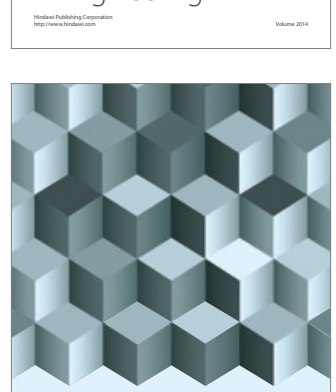

Journal of

Function Spaces
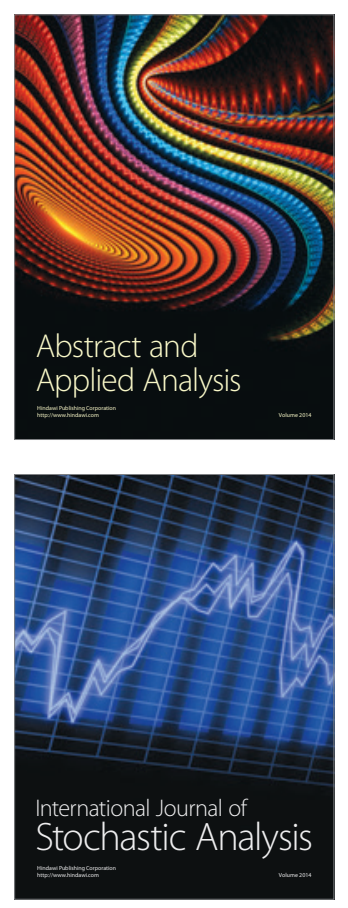

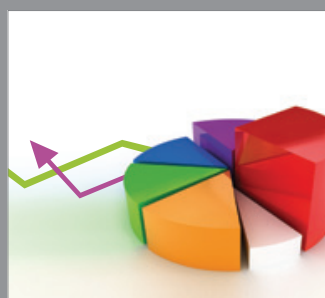

ournal of

Probability and Statistics

Promensencen
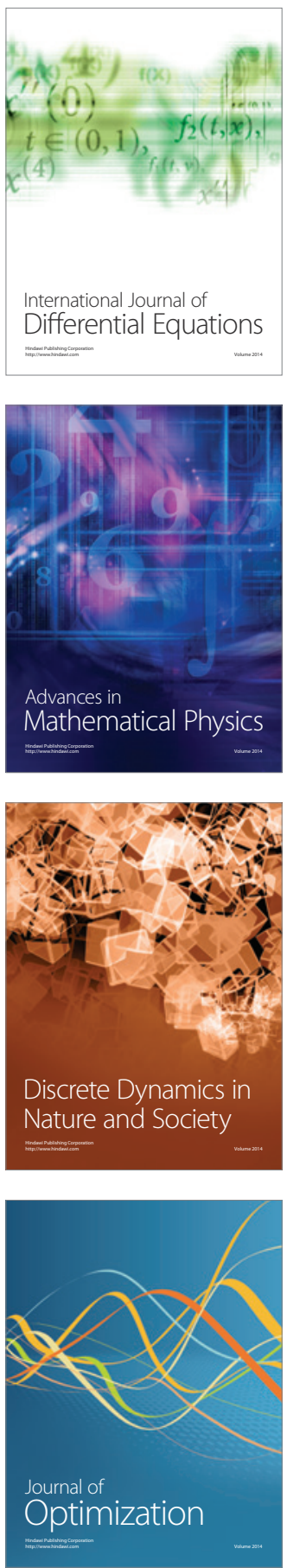